\title{
ALGORITMOS EVOLUTIVOS MULTIOBJETIVO APLICADOS EM SELEÇÃO DE CARACTERÍSTICAS
}

\author{
Velder Araujo Soares $^{1}$ e Fabiana Cristina Bertoni ${ }^{2}$ \\ 1. Bolsista PIBIC/CNPq, Graduando em Engenharia de Computação, Universidade Estadual de Feira de Santana, \\ e-mail: velder.vas@gmail.com \\ 2. Orientador, Departamento de Ciências Exatas, Universidade Estadual de Feira de Santana, e-mail: \\ fcbertoni@gmail.com
}

\author{
PALAVRAS-CHAVE: Algoritmos Evolutivos Multiobjetivo; Seleção de \\ características; Mineração de Dados.
}

\section{INTRODUÇÃO}

A seleção de características (ou redução da dimensionalidade) é um dos principais e mais utilizados métodos na mineração de dados. O objetivo da seleção de características é selecionar o subconjunto de características mais apropriado do conjunto de dados original, eliminando as características irrelevantes (Liu e Motoda 2002).

Técnicas de seleção de características não alteram a representação original das variáveis, mas apenas selecionam um subconjunto delas. Assim, elas preservam a semântica original das variáveis, oferecendo, portanto, a vantagem de interpretabilidade por um especialista sobre o domínio (Saeys et al., 2007). Apesar dos benefícios que a seleção de características provê, existe o problema do equilíbrio entre redução e precisão, onde se tenta manter a qualidade na classificação, minimizando a quantidade de dados.

$\mathrm{Na}$ busca do melhor equilíbrio entre redução dos dados e precisão, diversas metodologias vêm sendo propostas para selecionar características. No entanto, após o estudo dos trabalhos relacionados, notou-se que muitos utilizaram algoritmos evolutivos para seleção de características, mas nenhum deles é um Algoritmo Evolutivo Multiobjetivo (AEMO), apesar de tais métodos buscarem otimizar dois objetivos considerados conflitantes entre si, precisão e redução do custo computacional.

Diante do exposto, este trabalho se propõe a avaliar a aplicação de três AEMO amplamente conhecidos pela comunidade científica, sendo eles, o Non-dominated Sorting Genetic Algorithm (NSGA-II) (Deb et al., 2002), o NSGA-III (Deb \& Jain, 2014) e o Non-Dominated Sorting Genetic Algorithm Distance Oriented (NSGA-DO) (Pimenta \& Camargo, 2015) na seleção de características em bases de dados, além de validar a efetividade do método por meio de testes estatísticos.

\section{METODOLOGIA}

Para selecionar características, foram aplicados três AEMO, utilizando o framework jMetal (Durillo \& Nebro, 2011), sendo eles: NSGA-II, NSGA-III e o NSGA-DO. O Problema foi modelado considerando uma codificação binária do cromossomo, onde 1 indica a presença de uma característica e 0 sua ausência. Os operadores escolhidos juntamente com suas taxas para cruzamento, mutação e seleção foram Single Point 
Crossover (0.9), Bit Flip Mutation (0.2) e Binary Tournament, respectivamente. O tamanho da população foi definido em 100 indivíduos e como critério de parada, foram consideradas 100 gerações.

A função de fitness é responsável por avaliar cada cromossomo a partir dos dois objetivos estabelecidos, acurácia e taxa de redução, que são obtidos através das equações 1 e 2 respectivamente.

$$
\begin{gathered}
\text { acurácia }=\frac{\text { total de acertos }}{\text { total caracteristicas }} \\
\text { taxa de redução }=\frac{\text { total carac. - total carac reduzidas }}{\text { total características }}
\end{gathered}
$$

$\mathrm{Na}$ Equação 1, o número total de acertos é proveniente da aplicação do classificador $k$-Nearest Neighbor (KNN) disponível no WEKA ${ }^{1}$, com o valor de $K$ igual a 5 . Uma vez que o valor de $\mathrm{K}$ pode variar de uma base de dados para outra, pois não há conhecimento de novas metodologias para a escolha absoluta do mesmo, além de tentativa e erro (Dreiseitl \& Ohno-Machado, 2002). Neste estudo, o valor de K foi definido a partir de experimentos, que utilizaram os valores 1,3 e 5 .

Após a aplicação de cada AEMO na seleção das características, foi utilizado como classificador para os testes, o Support Vector Machine (SVM), também disponível no WEKA, que se baseia na teoria de aprendizado estatístico desenvolvida por (Vapnik, 2013). O SVM constrói ótimos limites de separação de um determinado conjunto de dados por meio de um hiperplano, tendo desempenhos iguais ou superior a outros classificadores em estudos na literatura médica (Dreiseitl \& Ohno-Machado, 2002).

Para os experimentos, foram utilizadas nove bases de dados extraídas do repositório UCI Machine Learning ${ }^{2}$, todas consideradas grandes, por possuírem mais de 100 características. A Tabela 1 mostra as informações referentes a essas bases de dados.

Tabela 1. Bases de dados utilizadas na seleção de características

\begin{tabular}{lccc}
\multicolumn{1}{c}{ Dataset } & Instâncias & Atributos & Classes \\
\hline Arrhythmia & 452 & 279 & 13 \\
Dexter & 600 & 20000 & 2 \\
Gina Agnostic & 3468 & 970 & 2 \\
Hiva Agnostic & 4229 & 1617 & 2 \\
Isolet & 7797 & 617 & 26 \\
Madelon & 2600 & 500 & 2 \\
Mfeat-factors & 2000 & 216 & 10 \\
Mfeat-pixels & 2000 & 240 & 10 \\
Musk & 6598 & 166 & 2 \\
\hline
\end{tabular}

\footnotetext{
${ }^{1}$ WEKA: Waikato Environment for Knowledge Analysis

${ }^{2} \mathrm{https}$ ://archive.ics.uci.edu/ml/datasets.html
} 
As bases de dados originais foram divididas em conjuntos de treinamento e teste, utilizando a abordagem 10-fold cross validation (Kohavi, 1995). Além disso, cada fold foi testado três vezes, logo, para cada base de dados foram efetuadas 30 execuções. Portanto, os resultados expressam a média destas 30 execuções.

Para comparar os resultados obtidos antes e após a aplicação dos três AEMO, inicialmente foi utilizado o teste de Friedman (Friedman, 1940) para verificar se existem diferenças significantes entre os resultados. Uma vez percebida uma diferença significante, os resultados são comparados em pares, utilizando o teste Wilcoxon Signed-Ranks (Wilcoxon, 1945).

\section{RESULTADOS E DISCUSSÃO}

A Tabela 2 mostra a média das 30 execuções obtidas através do classificador SVM, aplicado nas bases originais e nas bases após as seleções de características, realizadas pelos três AEMO. As colunas AC e TR indicam acurácia e taxa de redução, respectivamente, e os valores são expressos em porcentagem.

Tabela 2. Resultado da classificação das bases de dados utilizando os três AEMO para selecionar características.

\begin{tabular}{lcccccccc}
\multirow{2}{*}{ Dataset } & \multicolumn{2}{c}{ Original } & \multicolumn{2}{c}{ NSGA-II } & \multicolumn{2}{c}{ NSGA-III } & \multicolumn{2}{c}{ NSGA-DO } \\
\cline { 2 - 4 } & AC & AC & TR & AC & TR & AC & TR \\
\hline Arrhythmia & 70.60 & 69.86 & 58.75 & 68.74 & 57.40 & 70.29 & 58.66 \\
Dexter & 92.83 & 89.06 & 51.09 & 89.94 & 50.85 & 90.22 & 51.14 \\
Gina Agnostic & 83.74 & 85.12 & 54.64 & 85.02 & 53.44 & 85.34 & 54.22 \\
Hiva Agnostic & 94.28 & 95.02 & 53.43 & 94.82 & 52.65 & 94.99 & 53.48 \\
Isolet & 96.84 & 95.65 & 55.12 & 95.76 & 53.21 & 95.60 & 55.00 \\
Madelon & 55.96 & 57.69 & 56.70 & 56.33 & 54.79 & 57.10 & 56.45 \\
Mfeat-factors & 99.85 & 99.65 & 63.84 & 99.63 & 60.08 & 99.58 & 63.56 \\
Mfeat-pixels & 99.35 & 99.25 & 62.03 & 99.05 & 58.94 & 99.10 & 61.86 \\
Musk & 100.00 & 99.34 & 64.87 & 99.75 & 60.87 & 100.00 & 64.22 \\
\hline
\end{tabular}

De acordo com os resultados apresentados na Tabela 2, os métodos propostos apresentaram pequenas variações na precisão do classificador em comparação com as bases de dados originais. Para checar se essas variações têm alguma relevância, o teste de Friedman foi aplicado utilizando um nível de confiança $\alpha=0,05$, resultando num $p$-value $=0,36$. Isso implica que não há diferenças estatísticas entre os resultados em relação a acurácia.

Desse modo, a redução das bases de dados em mais de $50 \%$ em todos os datasets, utilizando três diferentes AEMO, não comprometeu o desempenho do classificador SVM.

\section{CONSIDERAÇÕES FINAIS}


Neste trabalho realizou-se um estudo na aplicação de três algoritmos evolutivos multiobjetivo na seleção de características em nove bases de dados. Também foi realizada uma comparação estatística dos valores de acurácia, considerando as bases de dados originais e reduzidas, em relação a precisão do classificador SVM.

Os resultados mostraram que todas as bases de dados foram reduzidas em aproximadamente $50 \%$ e que apesar da redução, o desempenho do classificador SVM não foi prejudicado de acordo com o teste de Friedman. Desse modo, a seleção de características por meio de algoritmos evolutivos multiobjetivo, torna-se uma alternativa para se obter um subconjunto reduzido de características com o mesmo desempenho do conjunto original.

\section{REFERÊNCIAS}

LIU, H., MOTODA, H.. On Issues of Instance Selection. Data Mining and Knowledge Discovery, vol.6, n.2, p. 115-130, 2002.

SAEYS, Yvan; INZA, Iñaki; LARRAÑAGA, Pedro. A review of feature selection techniques in bioinformatics. bioinformatics, v. 23, n. 19, p. 2507-2517, 2007.

DEB, Kalyanmoy et al. A fast and elitist multiobjective genetic algorithm: NSGA-II. IEEE transactions on evolutionary computation, v. 6, n. 2, p. 182-197, 2002.

DEB, Kalyanmoy; JAIN, Himanshu. An evolutionary many-objective optimization algorithm using reference-point-based nondominated sorting approach, part I: Solving problems with box constraints. IEEE Trans. Evolutionary Computation, v. 18, n. 4, p. 577-601, 2014.

PIMENTA, Adinovam HM; DE ARRUDA CAMARGO, Heloisa. NSGA-DO: Non-Dominated Sorting Genetic Algorithm Distance Oriented. In: Fuzzy Systems (FUZZ-IEEE), 2015 IEEE International Conference on. IEEE, 2015. p. 1-8.

KOHAVI, Ron et al. A study of cross-validation and bootstrap for accuracy estimation and model selection. In: Ijcai. 1995. p. 1137-1145.

DURILLO, Juan J.; NEBRO, Antonio J. jMetal: A Java framework for multi-objective optimization. Advances in Engineering Software, v. 42, n. 10, p. 760-771, 2011.

HOLMES, Geoffrey; DONKIN, Andrew; WITTEN, Ian H. Weka: A machine learning workbench. In: Intelligent Information Systems, 1994. Proceedings of the 1994 Second Australian and New Zealand Conference on. IEEE, 1994. p. 357-361.

DREISEITL, Stephan; OHNO-MACHADO, Lucila. Logistic regression and artificial neural network classification models: a methodology review. Journal of biomedical informatics, v. 35, n. 5-6, p. 352-359, 2002.

VAPNIK, Vladimir. The nature of statistical learning theory. Springer science \& business media, 2013.

FRIEDMAN, Milton. A comparison of alternative tests of significance for the problem of m rankings. The Annals of Mathematical Statistics, v. 11, n. 1, p. 86-92, 1940.

WILCOXON, Frank. Individual comparisons by ranking methods. Biometrics bulletin, v. 1, n. 6, p. 80-83, 1945. 